\title{
Computation of the Spitzer function in stellarators and tokamaks with finite collisionality
}

\author{
Winfried Kernbichler ${ }^{1}$, a , Gernot Kapper ${ }^{1}$, Sergei V. Kasilov ${ }^{1,2}$, and Nikolai B. Marushchenko ${ }^{3}$ \\ ${ }^{1}$ Fusion@ÖAW, Institute for Theoretical and Computational Physics, TU Graz, Austria \\ ${ }^{2}$ Institute of Plasma Physics, National Science Center "Kharkov Institute of Physics and Technology", Ukraine \\ ${ }^{3}$ Max Planck Institute for Plasma Physics, EURATOM Association, Greifswald, Germany
}

\begin{abstract}
The generalized Spitzer function, which determines the current drive efficiency in tokamaks and stellarators is modelled for finite plasma collisionality with help of the drift kinetic equation solver NEO-2 [1]. The effect of finite collisionality on the global ECCD efficiency in a tokamak is studied using results of the code NEO-2 as input to the ray tracing code TRAVIS [2]. As it is known [3], specific features of the generalized Spitzer function, which are absent in asymptotic (collisionless or highly collisional) regimes result in current drive from a symmetric microwave spectrum with respect to parallel wave numbers. Due to this effect the direction of the current may become independent of the microwave beam launch angle in advanced ECCD scenarii (O2 and X3) where due to relatively low optical depth a significant amount of power is absorbed by trapped particles.
\end{abstract}

\section{Introduction}

The generalized Spitzer function is an important part of current drive calculations within the adjoint approach [4] where it plays the role of current drive efficiency in phase space. This function is well studied and is effectively computed in asymptotical regimes with high and low collisionality (short and long mean free path regimes). In these regimes, the dimension of the kinetic equation does not exceed two because the generalized Spitzer function has a parametric dependence on spatial coordinates (in the long mean free path regime it essentially depends on the integrals of motion in velocity space only). In regimes with finite plasma collisionality the dependence of this function on the position on the flux surface is not trivial anymore. Its computation on a given flux surface requires the solution of a $3 \mathrm{D}(4 \mathrm{D})$ problem in tokamak (stellarator) geometry. This is a rather demanding task. Often it is performed using model simplifications of the kinetic equation, in particular, of the collision model. In the present report, a parallel version of the kinetic equation solver NEO-2 [1] is used to solve the Spitzer problem in stellarator geometry. This code does not employ any simplifying assumptions for the collision operator or for the device geometry. Prior to the parallelization of the code, a sequential NEO-2 version was mainly used for computation of mono-energetic neoclassical transport coefficients in stellarators [5] and for computation of the generalized Spitzer function in tokamaks [6], while computa-

a. e-mail: winfried.kernbichler@tugraz.at tions of this function for stellarators required rather long computing times.

Specific features of the global ECCD efficiency resulting from finite plasma collisionality are studied here with help of the ray tracing code TRAVIS [2] using the Spitzer function pre-computed by the code NEO-2. In contrast to asymptotical regimes where the Spitzer function is an odd function of parallel velocity, it does not have a distinct parity in finite collisionality regimes. This makes the generation of current by waves with symmetric spectra over parallel wave numbers [3] possible. As shown below, this effect may become dominant in ECCD scenarii with relatively weak absorption, such as O2 and X3, where a significant power fraction is absorbed in the trapped particle region of velocity space.

\section{ECCD computations in the adjoint approach}

The standard method for calculation of ECCD generated current in tokamaks and stellarators is the adjoint approach where the solution to a linearized quasilinear kinetic equation written in terms of the integrals of motion in velocity space and ignoring the cross field drift,

$$
v_{\|} \mathbf{h} \cdot \nabla \tilde{f}-\hat{L}_{C L} \tilde{f}=Q_{R F},
$$

is reduced to a generalized Spitzer problem. Here, $\tilde{f}$ is the perturbation of the electron distribution function, $\mathbf{h}=\mathbf{B} / B$ is a unit vector along the magnetic field, $v_{\|}$ 
is the parallel velocity, $\hat{L}_{C L}$ is the linearized collision integral and

$$
Q_{R F}=-\frac{\partial}{\partial \mathbf{p}} \cdot \boldsymbol{\Gamma}_{R F}=\hat{L}_{Q L} f_{M}
$$

is the quasilinear particle source in phase space resulting from the action of the quasilinear diffusion operator $\hat{L}_{Q L}$ on the Maxwellian $f_{M}$. Using the adjoint approach (see e.g., Ref. [4]), the flux surface averaged parallel current density is expressed through the adjoint generalized Spitzer function $\bar{g}$ (current drive efficiency) as follows,

$$
\left\langle j_{\|} b\right\rangle=\left\langle e b \int \mathrm{d}^{3} p v_{\|} \tilde{f}\right\rangle=e l_{c}\left\langle\int \mathrm{d}^{3} p \bar{g} Q_{R F}\right\rangle,
$$

where $\langle\ldots\rangle$ denotes "flux surface average" (average over the volume between neighboring flux surfaces), $b$ is the normalized magnetic field module, $e$ is the electron charge, $l_{c}$ is the free path length given by $l_{c}=T_{e}^{2} /\left(\pi n_{e} e^{4} \Lambda\right)$ where $n_{e}, T_{e}$ and $\Lambda$ are electron density, temperature and Coulomb logarithm, respectively. The adjoint function $\bar{g}$ in (3) is expressed through the generalized Spitzer function $g$ as follows, $\bar{g}\left(v_{\|}\right)=-g\left(-v_{\|}\right)$, where $g\left(v_{\|}\right)$is the solution to the conductivity problem,

$$
v_{\|} \mathbf{h} \cdot \nabla f_{M} g-\hat{L}_{C L} f_{M} g=\frac{b}{l_{c}} v_{\|} f_{M}
$$

In the homogeneous magnetic field or in the high collisionality limit the function $g$ is factorized to $g=b \lambda D$ where $\lambda=p_{\|} / p$ is the pitch parameter and $D=D(p)$ is the classical Spitzer function [7]. With the help of (2), the parallel current density is expressed via the momentum space flux density due to the waveinduced quasilinear diffusion, $\boldsymbol{\Gamma}_{R F}$, and the derivatives of the adjoint Spitzer function,

$$
\left\langle j_{\|} b\right\rangle=e l_{c}\left\langle\int \mathrm{d}^{3} p \frac{\partial \bar{g}}{\partial \mathbf{p}} \cdot \boldsymbol{\Gamma}_{R F}\right\rangle .
$$

Within geometrical optics used for calculation of $\mathrm{ECRH} / \mathrm{ECCD}$, the quasilinear flux density is described in a local approximation. In this approximation $\boldsymbol{\Gamma}_{R F}$ differs from zero in velocity space only at the resonance line where the (multiple) cyclotron resonance condition taking into account Doppler shift is fulfilled, $\omega=n \omega_{c}+k_{\|} v_{\|}$, where $\omega, \omega_{c}, n$ and $k_{\|}$are wave frequency, relativistic cyclotron frequency, cyclotron harmonic index and parallel wave vector, respectively. For weakly relativistic electrons the largest component of the quasilinear flux density is over perpendicular momentum. Therefore, as follows from (5), the behavior of the derivative of $\bar{g}$ over perpendicular momentum at the resonance curve is of main importance for ECCD.

\section{Solution of the generalized Spitzer problem in toroidal geometry}

In general toroidal geometry described in terms of flux coordinates $(s, \vartheta, \varphi)$, the dimension of the generalized
Spitzer problem (4) is four (flux surface label $s$ plays the role of a parameter). Within a field line integration technique used in the NEO-2 code this dimension is reduced by one because $g$ is represented on the flux surface by its values on a single field line, which is long enough to cover this flux surface densely. When presenting the dependence of the generalized Spitzer function on kinetic energy in the form of an expansion over the associated Laguerre polynomials of the order $3 / 2$ (Sonine polynomials),

$$
g(\mathbf{r}, \mathbf{p})=\sum_{m=0}^{M} g_{m}\left(\varphi_{s}, \eta\right) L_{m}^{(3 / 2)}\left(u^{2}\right),
$$

the problem is reduced to a set of coupled $2 \mathrm{D}$ equations for the expansion coefficients $g_{m}\left(\varphi_{s}, \eta\right)$, where $\varphi_{s}, \eta=p_{\perp}^{2} /\left(p^{2} B\right)$ and $u=p\left(2 m_{e} T_{e}\right)^{-1 / 2}=v v_{T}^{-1}$ are the toroidal angle playing the role of a field line parameter, the normalized perpendicular adiabatic invariant and the normalized momentum (normalized velocity) module, respectively. These equations are solved using a conservative finite difference scheme on a $2 \mathrm{D}$ grid adapted over $\eta$ in order to resolve the boundary layers $\eta \approx \eta_{b}$ associated in the long mean free path regime with local magnetic field maxima, $\eta_{b}=1 / B_{\max }$.

Due to the toroidal symmetry of a tokamak, the problem is periodic there with respect to $\varphi_{s}$ (see the lower plot in Fig. 1), and its solution (for a single field period) can be effectively computed by a single processor unit. This periodicity is missing in a stellarator (see Fig. 2) where it has to be solved for the whole field line. Such a solution is obtained in terms of Green functions ("propagators") introduced for separate field line segments between the local maxima ("ripples") with individual discretization over $\eta$. Propagators are then matched with each other using re-discretization. The computation of propagators has been parallelized recently what makes the evaluation of the Spitzer function in stellarators feasible on modern computer clusters.

In Fig. 1 the generalized Spitzer function in a tokamak is shown together with its derivative over perpendicular velocity for a finite collisionality case, $\bar{\nu}=0.01\left(\nu^{*}=0.22\right)$, and for the long mean free path limit computed by the code SYNCH [8]. Here $\bar{\nu}=4 \pi^{1 / 2} R /\left(3 l_{c}\right)$ is the universal collisionality parameter and $\nu^{*}=q \bar{\nu} Z_{\text {eff }}^{2} A^{3 / 2} \pi^{-1}$ is the conventional tokamak collisionality parameter, $R, q, A$ and $Z_{\text {eff }}$ are major radius, safety factor, aspect ratio, and effective charge number, respectively. It can be seen that the Spitzer function is strictly antisymmetric only at the magnetic field minimum and maximum points while for the points in between there is a significant symmetric part connected with the combined action of the magnetic mirroring force and collisional detrapping. This part becomes small in the long mean free path case where it is localized in the narrow trappedpassing boundary layer. The symmetric part of the generalized Spitzer function is responsible for current 

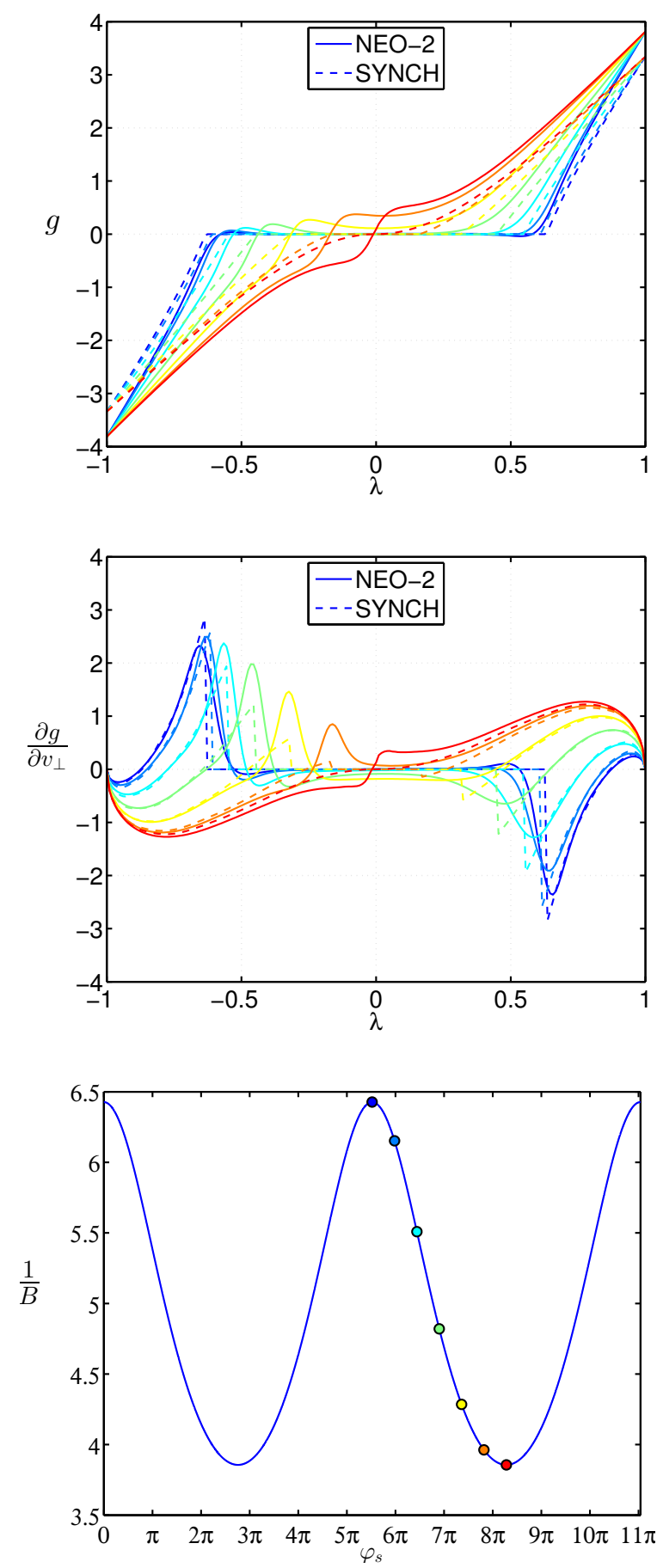

Figure 1. Generalized Spitzer function in a tokamak (upper plot) and its derivative over the normalized perpendicular velocity (middle plot) vs pitch parameter $\lambda=p_{\|} / p$ for the normalized velocity $u=2$ at various spatial points. Colors of the curves are the same as colors of the markers showing the values of the upper phase space boundary $\eta_{\max }=1 / B$ at respective observation points (lower plot). Solid and dashed curves correspond to the finite collisionality case and to the asymptotical low collisionality limit, respectively.
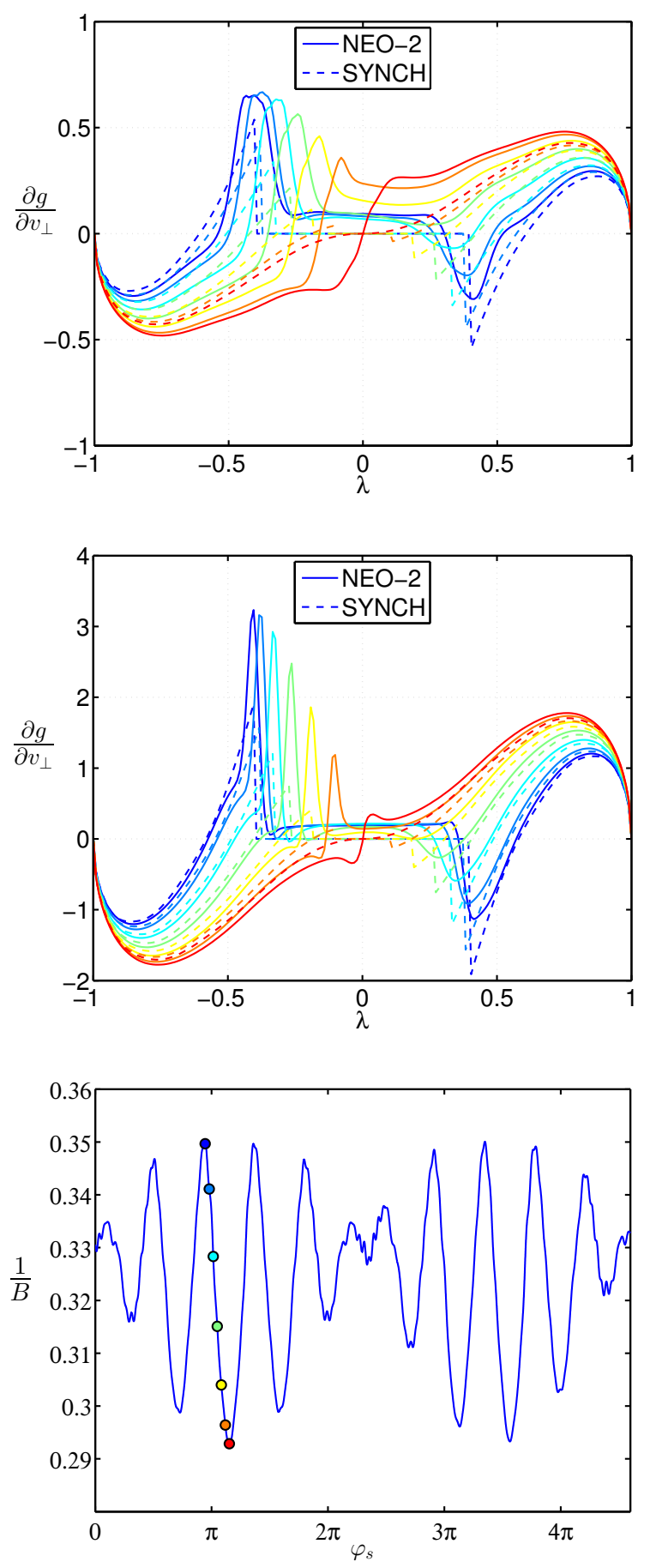

Figure 2. Derivative of the Spitzer function over the normalized perpendicular velocity in W7-X for $u=1$ (upper plot) and $u=2$ (lower plot). Colors and line styles are the same as in Fig. 1.

drive by waves with symmetric spectra [3], which result in a symmetric source term $Q_{R F}$ (this is similar to bootstrap current generation by the symmetric source term $-\mathbf{v}_{g} \cdot \nabla f_{M}$ with $\mathbf{v}_{g}$ being the cross-field drift velocity). Since the sign of this part is determined by the sign of the parallel derivative of the magnetic field module, the direction of the current is respectively determined by the location of the absorption zone in 

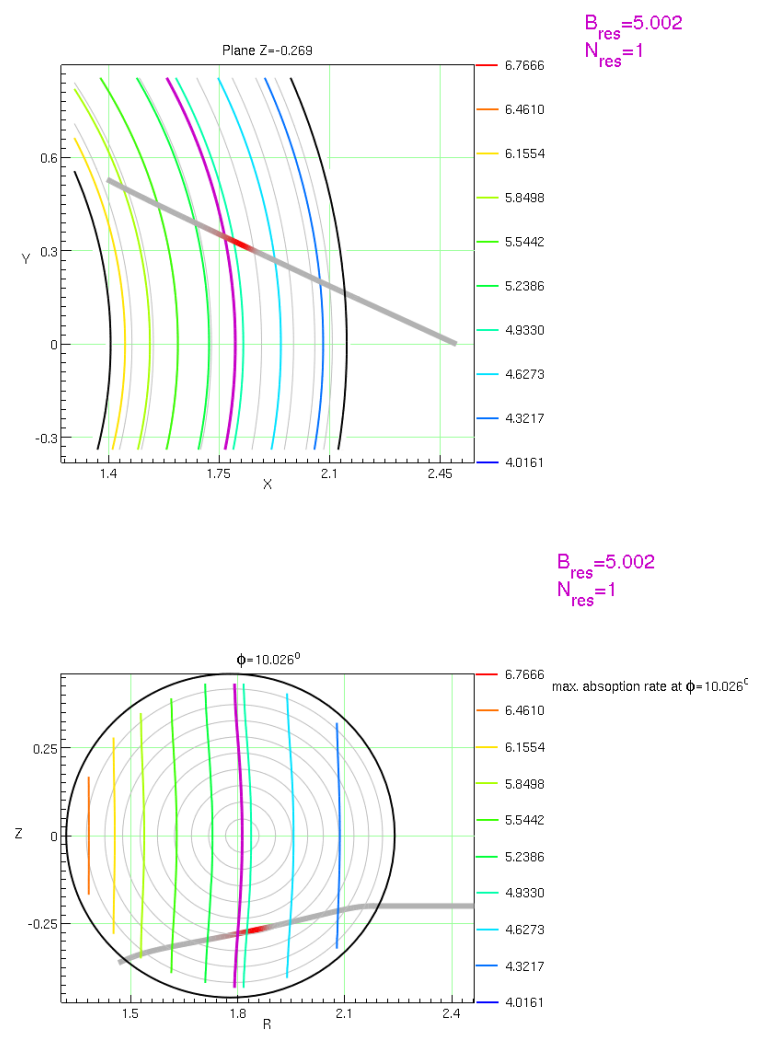

Figure 3. Ray trajectory (grey) in a tokamak for the toroidal launch angle $25^{\circ}$. Upper plot - view from top, lower plot - ray projection to the toroidal cross-section $\varphi=$ const (length units are SI). Cold resonance position is shown by the magenta line.

space. Behavior of the generalized Spitzer function in a stellarator (see Fig. 2 where its derivative is plotted for W7-X with $\bar{\nu}=0.01$ ) is similar to a tokamak except for the absence of the antisymmetry also in local extrema of the magnetic field along the field line.

\section{ECCD in a tokamak}

The impact of finite plasma collisionality on global ECCD efficiency has been studied in tokamak geometry (see Fig. 3) using the ray-tracing code TRAVIS [2] and various models for the generalized Spitzer function. In this example, parabolic density and temperature profiles are used. The values of magnetic field, central density and (homogeneous) $Z_{\text {eff }}$ are $5 \mathrm{~T}$, $10^{20} \mathrm{~m}^{-3}$ and 1.5 , respectively. Two central temperature values, $1 \mathrm{keV}$ and $2 \mathrm{keV}$ resulted at the absorption region (shown with red color of the ray in Fig. 3) in plasma collisionalities $\nu^{*}=0.28$ and $\nu^{*}=0.07$, respectively. The microwave beam is launched off axis, in the horizontal plane, varying the toroidal launch angle $\beta$ (angle between the central ray and the direction to the tokamak symmetry axis).

Scans of the absorbed power fraction and total generated current over $\beta$ are shown for the standard ECCD
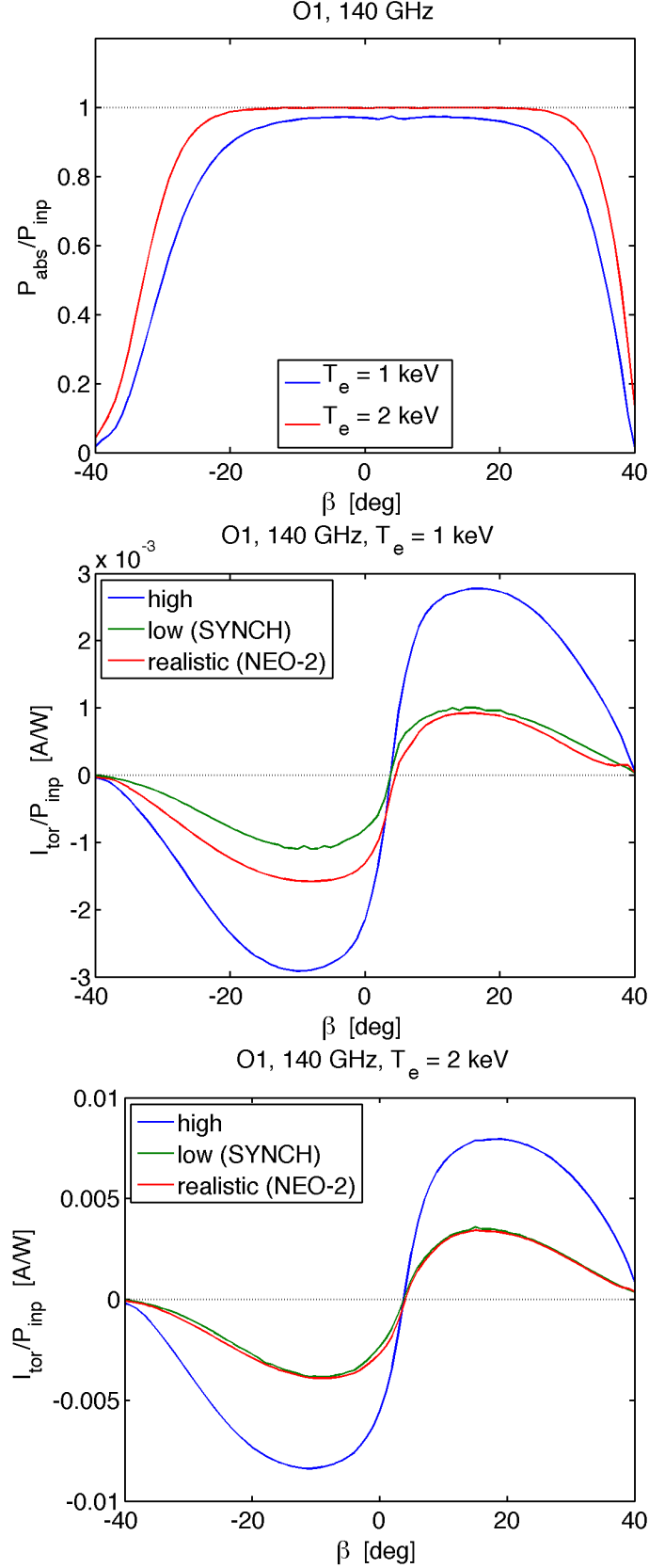

Figure 4. O1 resonance: Absorbed power fraction (upper plot) and total generated toroidal current for central electron temperature $1 \mathrm{keV}$ (middle plot) and $2 \mathrm{keV}$ (lower plot) as functions of the toroidal launch angle $\beta$. High, low and realistic collisionality models for the generalized Spitzer function are indicated in the legend.

scenarii employing the fundamental resonance for the ordinary mode (O1) and the second harmonic resonance for the extraordinary mode (X2) in Figs. 4 and 5, respectively. In these scenarii wave absorption is strong, and power is absorbed mainly by strongly passing particles from the high energy tail of the Maxwellian. Since the finite collisionality effect on the Spitzer function is not significant in this velocity space region (see Figs. 1 and 2), finite plasma collisionality does not principally modify the collision- 

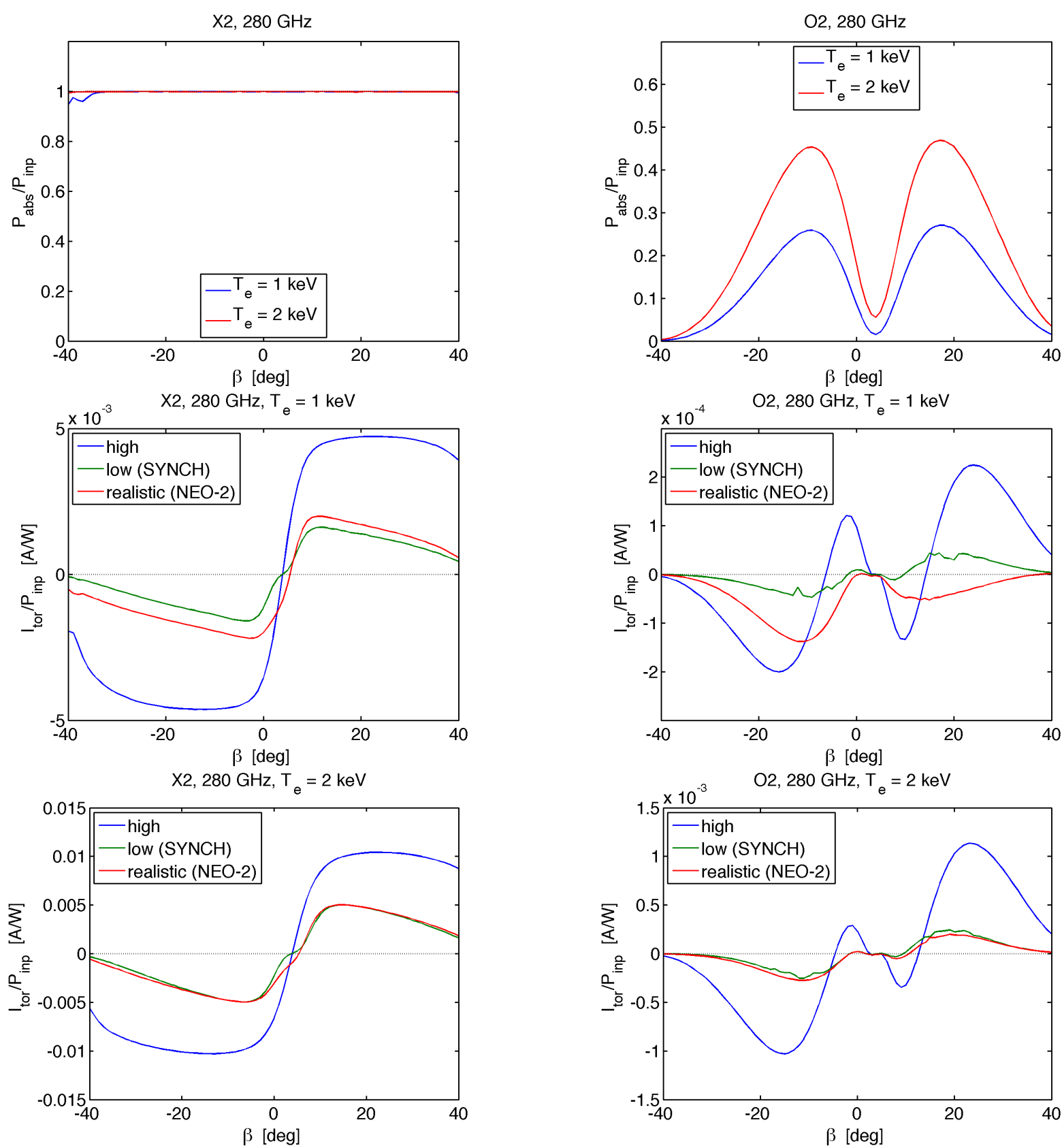

Figure 5. The same as in Fig. 4 for the X2 resonance.

less result. The impact of finite collisionality on the current is more significant for the advanced scenarii, which are the second harmonic resonance for the ordinary mode (O2) and the third harmonic resonance for the extraordinary mode (X3), as shown in Figs. 6 and 7 , respectively. These scenarii are characterized by a rather weak absorption, such that a significant amount of power is absorbed around the "cold" resonance by particles with relatively small parallel velocities and large perpendicular velocities (large Larmor radii). These particles belong to the velocity space region where the contribution of the symmetric part of the Spitzer function to the current can be dominant. As a result, at the onset of the banana regime $\left(\nu^{*}=0.28\right)$, the direction of the current becomes inde-

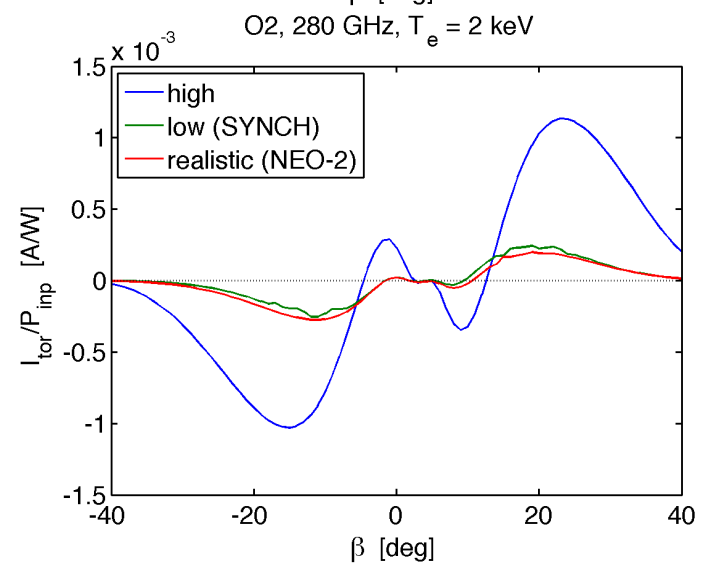

Figure 6. The same as in Fig. 4 for the $\mathrm{O} 2$ resonance.

pendent of the launch angle. This effect is absent in both, low and high collisionality cases and, therefore, it can not be computed at intermediate collisionalities if one uses for the Spitzer function a superposition of results from the asymptotical limits.

\section{Conclusion}

The finite collisionality effect on the Spitzer function is clearly important in toroidal plasmas, which are in the plateau regime or at the beginning of the long mean free path (banana) regime. In these regimes, the symmetric part of the Spitzer function localized around the trapped particle region is comparable with the antisymmetric part. This is in contrast to the 

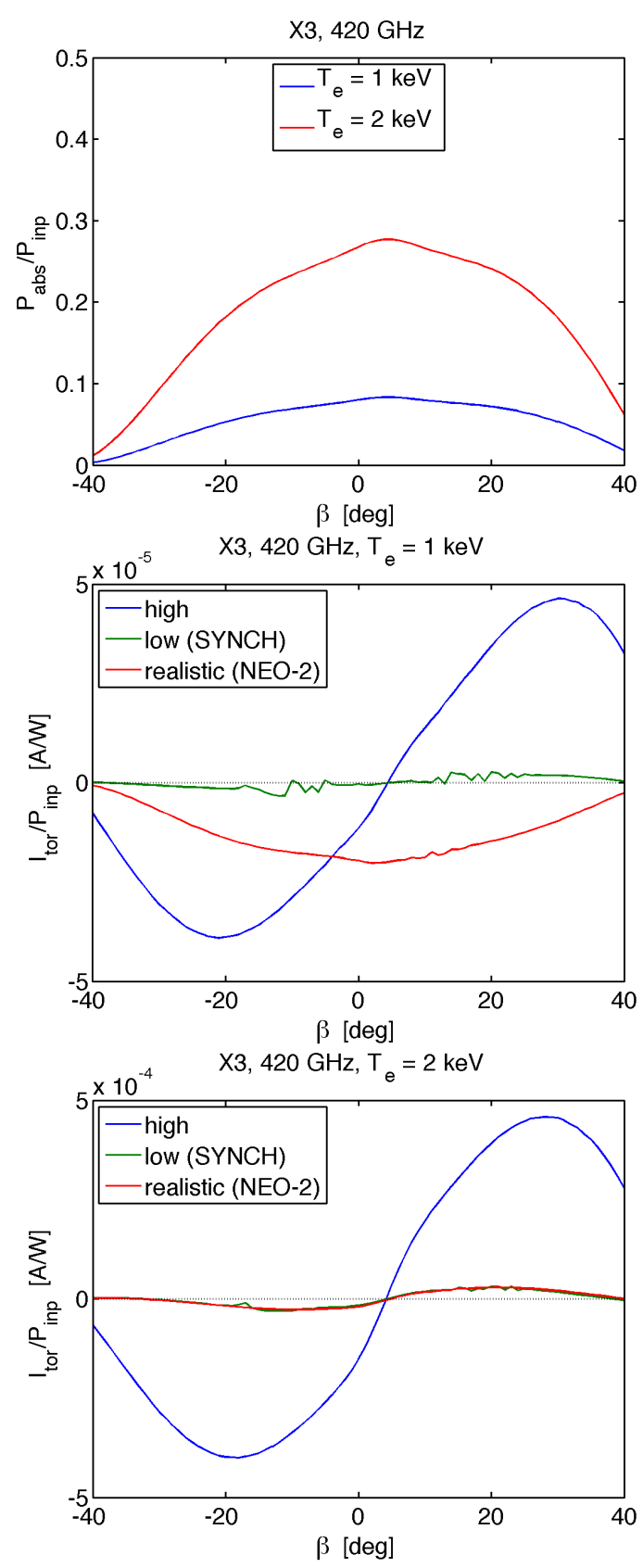

Figure 7. The same as in Fig. 4 for the $\mathrm{X} 3$ resonance. asymptotical low and high collisionality limits where the symmetric part is small. As a result, the direction of the current may become independent of the microwave beam launch angle in advanced ECCD scenarii with relatively low single pass absorption such as $\mathrm{O} 2$ and X3. These scenarii are less efficient than standard scenarii (O1 and X2) but they might be necessary in high density plasmas where wave cut-off makes $\mathrm{O} 1$ and $\mathrm{X} 2$ resonances not accessible.

\section{Acknowledgment}

This work, supported in part by the European Commission under the contract of Associations between EURATOM and the Austrian Academy of Sciences, was carried out within the framework of the European Fusion Development Agreement. The views and opinions expressed herein do not necessarily reflect those of the European Commission.

\section{References}

[1] W. Kernbichler, et al, Journal of Plasma and Fusion Research 3, 51061 (2008)

[2] N. B. Marushchenko, et al., Fusion Sci. Technol. 50, 180 (2009)

[3] P. Helander, P. J. Catto, Phys. Plasmas 8, 1988 (2001)

[4] T. M. Antonsen, K. R. Chu, Phys. Fluids 25, 1295 (1982)

[5] C.D. Beidler, et al, Nuclear Fusion 51, 076001 (2011)

[6] W. Kernbichler, et al, Contributions to Plasma Physics 50, 761 (2010)

[7] L. Spitzer, R. Härm, Phys. Rev. 89, 977 (1953)

[8] S.V. Kasilov, W. Kernbichler, Phys. Plasmas 3, 4115 (1996) 